\title{
Single-Mode Spectral Beam Combining of High Power Tm-Doped Fiber Lasers with WDM cascades
}

\author{
Sinem Yilmaz ${ }^{* 1,3}$, Christoph Ottenhues ${ }^{3}$, Thomas Theeg $^{3}$, Samir Lamrini ${ }^{5}$, Karsten Scholle $^{5}$, \\ Michael Schäfer ${ }^{5}$, Peter Fuhrberg ${ }^{5}$, Hakan Sayinc ${ }^{3}$, Fatih Ömer Ilday ${ }^{1,2}$, Jörg Neumann ${ }^{3}$, Ludger \\ Overmeyer ${ }^{4}$ and Dietmar Kracht ${ }^{3}$ \\ ${ }^{1}$ Department of Physics, Bilkent University, Ankara, 06800, Turkey \\ ${ }^{2}$ Department of Electrical and Electronics Engineering, Bilkent University, Ankara, 06800, Turkey \\ ${ }^{3}$ Laser Zentrum Hannover e.V., Hollerithallee 8, 30419 Hannover, Germany \\ ${ }^{4}$ Institut für Transport und Automatisierungstechnik, An der Universität 2, 30823 Garbsen, Germany \\ ${ }^{5}$ Lisa Laser Products OHG, Max-Planck-Str 1,37191 Katlenburg-Lindau, Germany
}

\begin{abstract}
Spectral beam combining of Tm-doped fiber lasers can increase the laser output power while simultaneously maintaining the single mode beam quality. We report on a spectral beam combining technique based on highly efficient in-housemade WDM cascade. We demonstrate continuous wave power combining employing a WDM cascade consisting of four fiber laser sources with emission wavelengths of 1920, 1949, 1996 and $2030 \mathrm{~nm}$. A combined power of up to $38 \mathrm{~W}$ resulted in a combining efficiency of $69 \%$.
\end{abstract}

Keywords: Laser beam combining, Fiber Lasers, Thulium doped fiber lasers

\section{INTRODUCTION}

There has been a great interest in the development of high power fiber lasers in recent years as a result of the practicality of these lasers, along with the possibility to generate very high power with excellent beam quality that is ideal for applications throughout research, defense and industry. New directions are constantly being pursued to utilize the capabilities of this technology and one of these directions involves extending the emission wavelength further into the infrared range which will be needed for numerous existing and future applications ${ }^{1-3}$. Although the variety of rare-earth ion transitions and host materials have been tested for fiber laser emission in different wavelength regimes, a clear decrease of output power is observed at $\operatorname{Ref}(4)$ as plotting the maximum obtainable continuous wave output power of fiber lasers as a function of emitted wavelength. The primary reason of the decreasing behaviour of obtainable continuous wave output power is the growth in the quantum defect at longer wavelengths when the system is pumped with the traditional pump sources for silicate glass fiber lasers which are emitting light in the near infrared region close to $1 \mu \mathrm{m}$. The excellent cooling properties of optical fibers help to reduce the problem of high quantum defect, which creates heat that becomes an increasing fraction of the absorbed pump power. However, development of high power pump sources at longer wavelengths and selecting more appropriate laser transitions can be effectively used for diminishing the effects of high quantum defect and improving the laser output performance.

Therefore, scaling fiber lasers to high average output power is of current interest, especially at longer wavelengths ${ }^{5-9}$. Despite the easier engineering at longer wavelengths, owing to a number of scaling factors, scaling the power of single mode fiber lasers is still limited by optical or thermal damage of the fiber including thermal aberrations and nonlinear effects. Fundamental nonlinear and thermal limitations on the power scaling of fiber lasers which can be encountered by a single laser source are leading researchers into beam combining schemes to achieve higher continuous wave laser power. The goal of beam combining cannot simply be to increase the power, but to preserve the beam quality for applications in which high intensity is required on the target. The beam combination method will provide a path to even higher power with an excellent beam quality by using developed methods ${ }^{10,11}$ with the help of increasing the power level within each individual laser unit.

*s.yilmaz@1zh.de;

Fiber Lasers XIII: Technology, Systems, and Applications, edited by John Ballato, Proc. of SPIE Vol. 9728, 972800 - (c) 2016 SPIE · CCC code: 0277-786X/16/\$18 - doi: 10.1117/12.2212632 
A common approach for beam combining is to use a wavelength selective element to combine spectrally nonoverlapping laser sources. To date, almost all wavelengths combination systems having been demonstrated operate at 1 $\mu \mathrm{m}$ and $1.5 \mu \mathrm{m}$ bands. Also, despite the technical difficulties, beam combining of Thulium sources was demonstrated recently ${ }^{12}$. However, the beam quality of the laser sources degraded completely due to the use of combiners employing multimode fibers. Spectral beam combining utilizing single mode WDMs is a promising approach ${ }^{13}$, as the high beam quality of the laser building blocks is maintained ${ }^{14,15}$.

We report on spectral beam combining of four thulium-doped fiber oscillators by using a highly efficient in-housemade WDM cascade at a power level of $38 \mathrm{~W}$. The combining section consists of three WDMs, while two of them were used for combining oscillators with a small wavelength separation of $30 \mathrm{~nm}$, a third one was used to combine both wavelength sub-groups. Our approach enables further power scaling by increasing the output power of the distinct laser oscillators and by increasing the number of channels of the cascaded combination scheme of WDMs.

\section{EXPERIMENTAL SETUP}

The experimental design used in this work is sketched in Fig. 1. It contains the fiber laser sources and the spectral-beam combination scheme. As laser sources, four identical continuous wave oscillators were employed each emitting narrow line-width signals at distinct wavelengths, which are $1920 \mathrm{~nm}, 1949 \mathrm{~nm}, 1996 \mathrm{~nm}$, and $2030 \mathrm{~nm}$ with a respective output power of $7 \mathrm{~W}, 17 \mathrm{~W}, 20 \mathrm{~W}$ and $13 \mathrm{~W}$ as shown in Fig. 1. The cavity was entirely fiber-integrated, including pump delivery, which rendered the system misalignment free. The linear laser cavity comprised of a section of double-clad Tm-doped fiber, high-reflector fiber Bragg grating (FBG), a low-reflector FBG acting as output coupler, a pump combiner with a fiber integrated pump port and pump diodes up to a power of $50 \mathrm{~W}$. The schematic of the experimental setup is shown in Fig. 1.

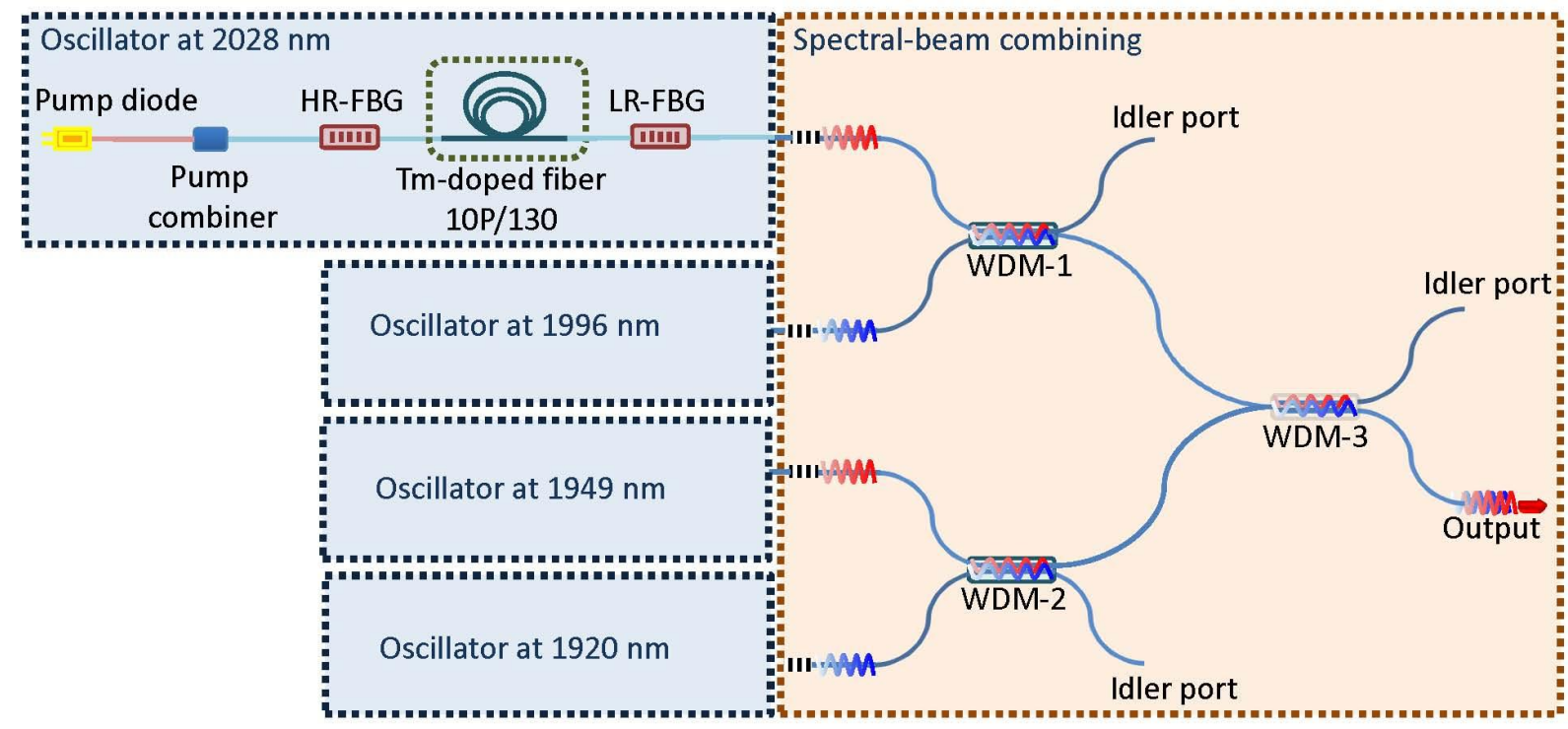

Figure 1. One of the continuous-wave fiber oscillator setups for spectral-beam combining and the schematic diagram of the spectral Beam-combining setup. HR-FBG: High reflective fiber-Bragg grating, LR-FBG: Low reflective fiber-Bragg grating, WDM:Wavelength-division multiplexer (WDM1 for combination of 1996 \& $2030 \mathrm{~nm}$, WDM2 for combination of 1920 \& 1949 $\mathrm{nm})$.

For the laser sources, two types of fibers were used. First one was the pump fiber which has $105 / 125 \mu \mathrm{m}$ core/cladding diameters. The other fiber type has $10 / 125 \mu \mathrm{m}$ DC core/cladding diameters featuring truly single mode operation. The active medium consisted of 5 meters of Tm-doped fiber with peak absorption of $3 \mathrm{~dB} / \mathrm{m}$ at a wavelength of $793 \mathrm{~nm}$. The length of the gain fiber was optimized by maximum attainable signal power. For pumping the system, fiber coupled pump diodes from DILAS were used emitting at a wavelength of $793 \mathrm{~nm}$. One of the FBGs was highly reflecting (99\%) and the other one was partially reflecting $(10 \%)$. The combining scheme foresaw a combination into two subgroups with in-house-made WDM1 and WDM2 (1920/1949 nm and 1996/2030 nm). These subgroups were then multiplexed with the in-house-made WDM3. 


\section{RESULTS}

\subsection{Manufacturing \& Characterization of WDMs}

The WDMs were fabricated by the fused biconical taper technique. Single mode fibers (SMF28) were employed, which had an NA of approximately 0.13 around a wavelength of $2 \mu \mathrm{m}$. While the core diameter was $10 \mu \mathrm{m}$, the cladding diameter was $125 \mu \mathrm{m}$. The used fibers had a pure silica cladding. We simulated light propagation in the fused fiber couplers with our FFT-based BPM algorithm ${ }^{16,17}$. The optical fibers were heated with a torch between two twisted points to cause the core-cladding mode coupling. Schematic deployment of the setup for the fabrication is explained in detail by Birks et. al. ${ }^{18}$. We modified the manufacturing process to achieve efficient WDMs for coupling the wavelength pairs 1920 and $1949 \mathrm{~nm}$ as well as 1996 and $2030 \mathrm{~nm}$. The developed WDMs were characterized in terms of insertion losses of the respective branches of the WDMs and spectral dependence of the coupling ratio.

\subsection{Fiber Laser Oscillators}

High power fiber lasers contained three major technologies, such as high quality active fibers, passive fiber components which can handle high power and bright pump diodes. With the aim of demonstrating a power combination employing the WDM cascade, we developed four identical Tm-doped single-mode continuous wave fiber lasers with an output power of $7 \mathrm{~W}, 17 \mathrm{~W}, 20 \mathrm{~W}$ and $13 \mathrm{~W}$ respectively, operating at central wavelengths of $1920 \mathrm{~nm}, 1949 \mathrm{~nm}, 1996 \mathrm{~nm}$, and $2030 \mathrm{~nm}$ which are defined as L1, L2, L3 and L4 for being easily understood the Fig. 2. The slope efficiency for each system was $>40 \%$ demonstrating cross relaxation ${ }^{19}$.

\subsection{Combining Two Wavelengths with WDM1 \& WDM2}

The laser outputs were spliced to the in-house-made WDMs for spatially overlapping the beams with differing wavelengths as shown in Fig. 1. First, the behavior of the WDM1 and WDM2 was investigated by measuring the output power at the respective signal ports separately. The measured output power as a function of the launched signal power for both of the lasers was shown in Fig. 2(c-d) respectively. The coupling efficiencies of WDM1 and WDM2 were higher than $84 \%$. The spectra of combined wavelength groups are depicted in the Fig. 2(a-b) for WDM1 and WDM2, respectively. For the spectral measurement setup, we used a neutral density filter to attenuate the output power of the WDMs due to the average power limitation of the spectrum analyzer. Although, this filter had particularly weak wavelength dependence, it caused the modifications of the spectral intensities during the spectrum measurements of the maximum signal output power.
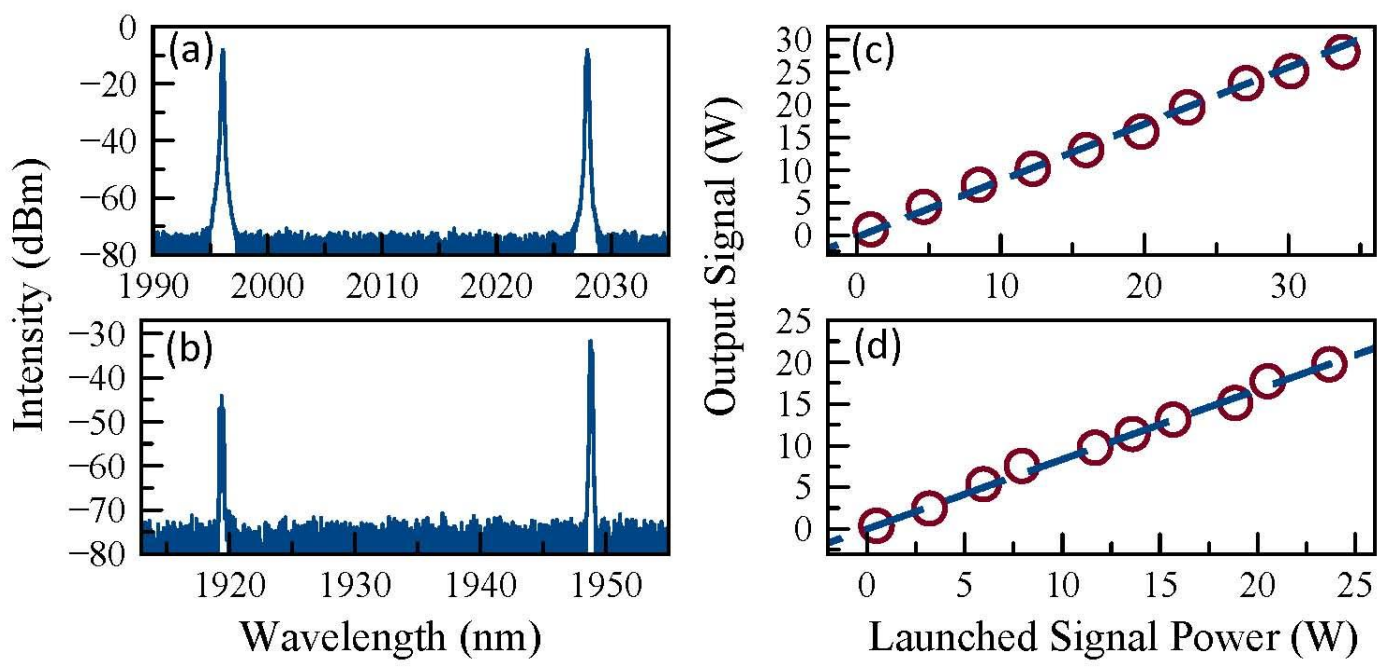

Figure 2. Spectral measurement at the signal port of (a) WDM2 and (b) WDM1. Combined optical power at the signal output port of the WDM2 (c) and WDM1 (d) with respect to the launched signal power for L3-L4 nm and L1-L2. 


\subsection{Combining Four Wavelengths with WDM3}

The setup for the combination of the two subgroups by the WDM3 is shown in Fig. 1. The spectrum measured at the output port of the WDM3 is shown in Fig. 3(a) and the combined laser power in dependence of the launched signal power is depicted in Fig 3(b). From Fig. 3(a), it is obvious that all wavelengths were successfully coupled to a single output port. We achieved more than $38 \mathrm{~W}$ of output power at a total input power of $48 \mathrm{~W}$, which results in a combining efficiency of $80 \%$. Two loss mechanisms can be identified for the combination of signals. The first one is the insertion loss of the WDMs and the second has its origin in the coupling of the signal power to the idler port which can be observed as a slow fluctuation of signal power at the output port. The error bars in the Fig. 3(b) represent this fluctuation.

A power of almost $5 \mathrm{~W}$ was coupled to the idler port of WDM3 at the maximum signal power level. This corresponds to $10 \%$ of the input power, and limits the coupling efficiency. The overall combining efficiency can be calculated to be around $69 \%$.
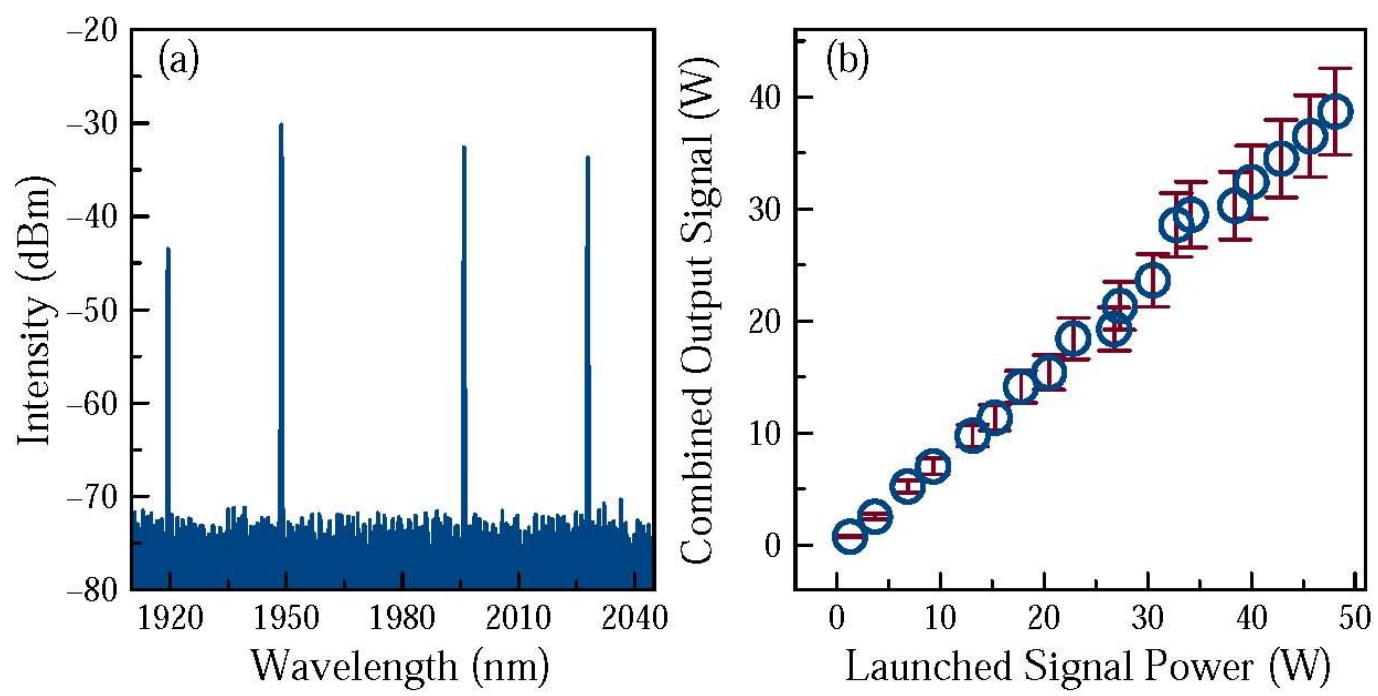

Figure 3. (a) Spectral power distribution at signal output port of WDM3. (b) Output power at signal output port of WDM3 with respect to input power.

\section{DISCUSSION}

Interest in spectrally beam combined laser systems is increasing rapidly, due to their simplicity and the high demand by applications which are not wavelength selective or which require the combination of distinct laser wavelengths. With our current implementation, we provide an all-fiber integrated design for the wavelength range of $2 \mu \mathrm{m}$ which is an alternative approach for obtaining not only flexibility and simplicity but also high output power with an excellent beam quality.

Due to the single mode fiber geometry most challenging limits are the onset of detrimental fiber nonlinearities like stimulated Brillouin scattering during the propagation of light after the oscillators in the fiber of the WDMs. In the weakly guiding approximation, the mode area of the lowest order mode scales as the square of the operation wavelength. This has a beneficial effect on the power scaling of fiber lasers at longer wavelengths. However, the drawback still arises from nonlinear effects that limit the maximum attainable output power after a threshold while maintaining the reliability for truly single mode high power operation. For the suppression of these effects at the higher power levels, distributing the power to distinct wavelengths is a promising approach for increasing the maximum attainable truly single mode output power ${ }^{20}$. 
The cascading concept offers maximum efficiency for largest possible separation of the wavelength groups and smallest possible separation of wavelengths within respective groups. Therefore, scaling up this configuration to an even higher power can also be expected by increasing the power per channel and increasing the number of channels which is related with the number of WDMs being used in the cascade design. Improvements on further power scaling by including additional channels can be achieved by combining a smaller spectral separation, which is limited by manufacturing parameters of WDMs. At the same time, the number of individual channels can be increased which is also related with largest possible separation of the wavelength groups inside the Tm-gain bandwidth of more than $100 \mathrm{~nm}$ around $1900 \mathrm{~nm}$. Hence, a further scaling by additional and a higher power per channel appears to be feasible in a straightforward manner.

\section{CONCLUSION}

In summary we demonstrated all-fiber truly single mode power combination of Tm-doped fiber lasers employing a WDM cascade. By using fiber-Bragg gratings as a highly reflective mirrors and output couplers, the distinct fiber lasers operated at wavelengths of 1920,1949, 1996 and $2030 \mathrm{~nm}$. The lasers were combined by using an in-house-made WDM cascade. The expected wavelength spacing and characteristics of WDMs can be freely specified based on central wavelengths of FBG pairs and the fiber lasers can be combined with changing the fabrication properties of the WDM. We achieved more than $38 \mathrm{~W}$ of output power from the signal port and the overall combining efficiency was measured to be $69 \%$. With a continuing improvement of the fiber laser output power and WDMs with high power handling capability, single mode operation at higher output power level can be achieved. The low quantum defect, the high spectral brightness, high average power pumping that can be provided by Thulium-doped fiber lasers enables efficient scaling of Holmium based amplifiers to ever higher average powers with great pulse energies. The demonstrated power combining scheme offers a new perspective for high brightness pump sources for laser operations and nonlinear effects in the MID-IR range.

\section{REFERENCES}

[1] Fried, N. M., and Murray, K. E., "High power thulium fiber laser ablation of urinary tissues at $1.94 \mu \mathrm{m}$," J. Endourol., 3, 25--31 (2005).

[2] Bach, T., Herrman, T. R., Cellarius, C., and Gross, A. J., "Bladder neck incision using a $70 \mathrm{~W}, 2 \mu \mathrm{m}$ continuous wave laser (RevoLix)," World J. Urol., 25, 263--267 (2007).

[3] Phillips, C. R., Edward, L. and Jiang, J., "Self-referenced frequency comb from a Tm-fiber amplifier via PPLN waveguide supercontinuum generation," Paper PDPA5 in CLEO:2011-Laser Applications to Photonics Applications, OSA Technical Digest (OSA, 2011).

[4] Jackson, S. D., "Towards high power mid-infrared emission from a fibre laser" Nature Photon. 6(7), 423--431 (2012).

[5] Ehrenreich, T., Leveille, R., Majid, I. and Tankala, K., "1-kW, All-glass Tm:fiber laser," SPIE Photonics West 2010:LASE, Fibre Lasers VII: Technology, Systems and Applications, Conference 7850 (2010).

[6] Hemming, A. V., Bennetts, S., Simakov, N., Haub, J. and Carter, A., "'Development of resonantly cladding-pumped holmium doped fiber lasers," Proc. SPIE 8237, 82371J (2012).

[7] Li, J., Hudson, D. D. and Jackson, S. D., "High power diode pumped fiber laser operating $3 \mu \mathrm{m}, "$ Opt. Lett, 36, 3642--3064 (2009).

[8] Carbonnier, C., Tobben, H. and Unrau, U. B., "Room temperature CW fibre laser at $3.22 \mu \mathrm{m}$," Electron. Lett., 34, 893--894 (1998).

[9] Schneider, J., Carbonnier, C. and Unrau, U.B., "Characterization of a $\mathrm{Ho}^{3+3}$ doped fluoride fiber laser with a $3.9 \mu \mathrm{m}$ emission wavelength, " Appl. Opt., 36, 8595--8600(1997).

[10] Wirth, C., Schmidt, O., Tsybin, I., Schreiber, T., Eberhardt, R., Limpert, J., Tünnermann, A., Ludewigt, K., Gowin, M., Have, E. T. and Jung, M. "High average power spectral beam combining of four fiber amplifiers to $8.2 \mathrm{~kW}$," Opt. Lett., 36(16), (2011).

[11] Shneider, O., Shulga, B. and Ishaaye, A. A., "Imposing narrow spectral bandwidth in a system of passively combined fiber lasers," Opt. Lett., 38(5), (2013). 
[12]Hemming, A. V., Simakov, N., Davidson, A., Bennetts, S., Hughes, M., Carmody, N., Davies, P., Corena, L., Stepanov, D., Haub, J., Swain, R. and Carter, A., "A Monolithic cladding pumped holmium-doped fibre laser," CLEO: Science and Innovation, San-Jose, California United States, ISBN:978-1-55752-972-5 (2013).

[13] Waarts, R. G., Welch, D. F., "High power marking system achieved through power scaling via multiplexing," US6411323 B1, 2002.

[14] Ottenhues, C., Theeg, T., Hausmann, K., Wysmolek, M., Sayinc, H., Neumann, J. and Kracht, D., “'Single-mode monolithic fiber laser with $200 \mathrm{~W}$ output power at a wavelength of $1018 \mathrm{~nm}$," Opt. Lett., 40(21), (2015).

[15] Theeg, T., Ottenhues, C., Sayinc, H., Neumann, J. and Kracht, D., " 158 W Core-pumped single frequency amplifier at kHz linewidth using a standard Ytterbium-doped single mode fiber," Photonics West 2015, 9344-6, (2015).

[16] Pelegrina-Bonilla, G., Hausmann, K., Liu, K., Sayinc, H., Morgner, U., Neumann, J. and Kracht, D. "Matching of the propagation constants in an asymmetric single-mode fused fiber coupler for core pumping thulium doped fiber at 795 nm," Opt. Lett., 37(11), 1844--1846(2012).

[17] Pelegrina-Bonilla, G., Hausmann, K., Tünnermann, H., Weßels, P., Sayinc, H., Morgner, U., Neumann, J. and Kracht, D., "Analysis of the Coupling Mechanism in Asymmetric Fused Fiber Couplers," IEEE J. Lightw. Technol., 32(13), (2014).

[18] Birks, T. A. and Li, Y. W., “'The shape of fiber tapers," J. Lightwave Technol., 10(4), 432--438 (1992).

[19] Jackson, S. D., "Cross relaxation and energy transfer up-conversion processes relevant to the functioning of $2 \mu \mathrm{m}$ $\mathrm{Tm}^{3+}$-doped silica fibre lasers," Opt. Commun., 230, 197-203 (2004).

[20] Dajani, I., Zeringue, C. and Shay, T. M., "Investigation of nonlinear effects in multitone-driven narrow-linewidth high-power amplifiers, " IEEE J. Sel. Topics Quantum Electron., 15(2), (2009). 